Cronfa - Swansea University Open Access Repository

This is an author produced version of a paper published in :

Medicine \& Science in Sports \& Exercise

Cronfa URL for this paper:

http://cronfa.swan.ac.uk/Record/cronfa21415

\title{
Paper:
}

MCNARRY, M., FARR, C., MIDDLEBROOKE, A., WELFORD, D., BREESE, B., ARMSTRONG, N. \& BARKER, A. (2015). Aerobic Function and Muscle Deoxygenation Dynamics during Ramp Exercise in Children. Medicine \& Science in Sports \& Exercise, 47(9), 1877-1884.

http://dx.doi.org/10.1249/MSS.0000000000000609

This article is brought to you by Swansea University. Any person downloading material is agreeing to abide by the terms of the repository licence. Authors are personally responsible for adhering to publisher restrictions or conditions. When uploading content they are required to comply with their publisher agreement and the SHERPA RoMEO database to judge whether or not it is copyright safe to add this version of the paper to this repository. http://www.swansea.ac.uk/iss/researchsupport/cronfa-support/ 


\section{AEROBIC FUNCTION AND MUSCLE DEOXYGENATION DYNAMICS DURING RAMP EXERCISE IN CHILDREN}

Melitta A. McNarry ${ }^{1}$, Colin Farr ${ }^{2}$, Andrew Middlebrooke ${ }^{3}$, Deborah Welford ${ }^{4}$, Brynmor Breese ${ }^{5}$, Neil Armstrong ${ }^{3}$ and Alan R. Barker ${ }^{3}$

${ }^{1}$ A-STEM, College of Engineering, Swansea University, Swansea, UK

${ }^{2}$ Medical Research Council Epidemiology Unit, Princes of Wales Hospital, Ely, UK.

${ }^{3}$ Children's Health and Exercise Research Centre, Sport and Health Sciences, College of Life and Environmental Sciences, University of Exeter, Exeter, UK

${ }^{4}$ Bishop Burton College, Beverly, UK

${ }^{5}$ Centre for Research in Translational Biomedicine, School of Biomedical and Healthcare

Sciences, Plymouth University, UK

Corresponding author:

Dr Alan R. Barker

Children's Health and Exercise Research Centre

Sport and Health Sciences

College of Life and Environmental Sciences

University of Exeter

St Luke's Campus

Exeter

EX1 2LU

Tel: 44 (0)1392 722766

Fax: 44 (0)1392 724726

Email: A.R.Barker@exeter.ac.uk 


\section{ABSTRACT}

Purpose: To characterise changes in deoxyhemoglobin ([HHb]) response dynamics in boys and girls during ramp incremental exercise to investigate whether the reduced peak oxygen uptake (peak $\dot{V} \mathrm{O}_{2}$ ) in girls is associated with a poorer matching of muscle $\mathrm{O}_{2}$ delivery to muscle $\mathrm{O}_{2}$ utilisation, as evidenced by a more rapid increase in $[\mathrm{HHb}]$.

Methods: 52 children ( 31 boys, $9.9 \pm 0.6$ years, $1.38 \pm 0.07 \mathrm{~m}, 31.70 \pm 5.78 \mathrm{~kg}$ ) completed ramp incremental exercise on a cycle ergometer during which pulmonary gas exchange and muscle oxygenation parameters were measured.

Results: When muscle [HHb] was expressed against absolute work rate and $\dot{V} \mathrm{O}_{2}$, girls had an earlier change in $[\mathrm{HHb}]$ as evidenced by the lower $c / d$ parameter (Girls: $54 \pm 20 \mathrm{~W}$ vs Boys: 67 $\pm 19 \mathrm{~W}, P=0.023$; Girls: $0.82 \pm 0.28 \mathrm{~L} \cdot \mathrm{min}^{-1}$ vs. Boys: $\left.0.95 \pm 0.19 \mathrm{~L} \cdot \mathrm{min}^{-1}, P=0.055\right)$ and plateau (Girls: $85 \pm 12 \mathrm{~W}$ vs. Boys: $99 \pm 18 \mathrm{~W}, P=0.031$; Girls: $1.02 \pm 0.25 \mathrm{~L} \cdot \mathrm{min}^{-1}$ vs. Boys: $\left.1.22 \pm 0.28 \mathrm{~L} \cdot \mathrm{min}^{-1}, P=0.014\right)$. However, when expressed against relative work-rate or $\dot{V} \mathrm{O}_{2}$, there were no sex differences in $[\mathrm{HHb}]$ response dynamics (all $P>0.20$ ). Significant correlations were observed between absolute and fat-free mass normalised peak $\dot{V} \mathrm{O}_{2}$ and the $\mathrm{HHb} c / d$ and plateau parameters when expressed against absolute work-rate or $\dot{V} \mathrm{O}_{2}$. Furthermore, when entered into a multiple regression model, the $[\mathrm{HHb}]$ plateau against absolute $\dot{V} \mathrm{O}_{2}$ contributed $12 \%$ of the variance in peak $\dot{V} \mathrm{O}_{2}$ after adjusting for fat-free mass, gas exchange threshold, and body fatness (model $\left.R^{2}=0.81, P<0.001\right)$.

Conclusion: The sex-difference in peak $\dot{V} \mathrm{O}_{2}$ in 9-10 year old children is, in part, related to sexspecific changes in muscle $\mathrm{O}_{2}$ extraction dynamics during incremental exercise.

Keywords: NIRS; $\mathrm{O}_{2}$ delivery; $\mathrm{O}_{2}$ utilization; peak $\dot{V} \mathrm{O}_{2}$; pre-pubertal; sex 


\section{INTRODUCTION}

2 A perplexing question in paediatric exercise physiology is the sexual dimorphism in peak oxygen

3 uptake $\left(\dot{V} \mathrm{O}_{2}\right)$ in pre-pubertal and pubertal children. Specifically, when normalised for body

4 mass, boys display a 10-15\% greater peak $\dot{V} \mathrm{O}_{2}$ compared to girls (3). This sex difference has

5 been attributed to changes in $\mathrm{O}_{2}$ delivery due to an elevated peak stroke volume in the presence

6 of a comparable peak heart rate resulting in a higher peak cardiac output in boys. However, when

7 stroke volume and cardiac output are normalised using fat free mass (FFM), the sex difference

8 for cardiac measures disappears (39). Consequently, scaling for FFM (39) or muscle volume (11,

9 40) reduces the sex difference in peak $\dot{V} \mathrm{O}_{2}$ to $<5 \%$. This has led to the notion that the higher

10 peak $\dot{V} \mathrm{O}_{2}$ in boys is predominantly related to their greater FFM.

12 This notion has recently been challenged, however, by Winsley et al. (43) who compared boys

13 and girls matched for FFM, and demonstrated a $\sim 15 \%$ higher peak $\dot{V} \mathrm{O}_{2}$ in boys, which was not

14 explained by differences in cardiac output, stroke volume or haemoglobin concentration. Rather,

15 a wider arterial mixed venous $\mathrm{O}_{2}$ content difference, estimated by rearrangement of the Fick

16 equation, was found in the boys, suggesting peripheral factors relating to the ability to deliver

17 and utilise $\mathrm{O}_{2}$ at the contracting muscle were the cause of the boys' higher peak $\dot{V} \mathrm{O}_{2}$. This

18 finding, however, contradicts studies showing no sex-differences in arterial mixed venous $\mathrm{O}_{2}$

19 content difference at maximal exercise in children $(29,39)$ and warrants further investigation.

21 Knowledge of changes in muscle $\mathrm{O}_{2}$ delivery and utilisation during incremental exercise in

22 children is largely limited to central measures of cardiac output, stroke volume and $\dot{V} \mathrm{O}_{2}$ which

23 may not faithfully reflect peripheral changes in the microcirculation (28). Microcirculatory 
24 changes in muscle $\mathrm{O}_{2}$ delivery and $\mathrm{O}_{2}$ utilisation can be obtained non-invasively using the near

25 infrared spectroscopy (NIRS) derived signal for muscle [deoxygenated haemoglobin and 26 myoglobin] ([HHb]) $(15,23)$. Rapid changes in $[\mathrm{HHb}]$ reflect an increase in fractional muscle $\mathrm{O}_{2}$

27 extraction, which is considered to reflect an inadequate matching of muscle $\mathrm{O}_{2}$ delivery to $\mathrm{O}_{2}$ 28 utilisation in the microcirculation. The increase in $[\mathrm{HHb}]$ during ramp exercise has been 29 characterised using a sigmoidal $(8,15,26)$ or bi-linear (37) model, and used to study the effect of 30 trained status and ageing $(8,18,26)$. Interestingly, the rate of change in $[\mathrm{HHb}]$ is more rapid in 31 adults $(8,18)$ and children (26) with a lower $\dot{V} \mathrm{O}_{2}$ max, indicating a greater rate of muscle $\mathrm{O}_{2}$ 32 extraction is required, presumably due to inadequate muscle $\mathrm{O}_{2}$ delivery. A recent study by 33 Murias et al. (27) examined the [HHb] response dynamics during ramp exercise in men and 34 women and found the latter to be characterised by a more rapid increase in [ $\mathrm{HHb}]$ and an earlier 35 plateau (i.e. attainment of maximal $\mathrm{O}_{2}$ extraction) when expressed relative to peak power and $36 \dot{V} \mathrm{O}_{2}$ max. This finding suggests that women have a poorer matching of muscle $\mathrm{O}_{2}$ delivery to $\mathrm{O}_{2}$ 37 utilisation during ramp exercise. In girls the rate of increase in [HHb] was recently shown to 38 correlate with peak $\dot{V} \mathrm{O}_{2}$ and the gas exchange threshold (GET) (26). However, it is currently 39 unknown whether similar sex-specific impairments in the matching of muscle $\mathrm{O}_{2}$ delivery to 40 utilisation during ramp exercise are present in children and whether this can explain, in part, the 41 sexual dimorphism in peak $\dot{V} \mathrm{O}_{2}$.

43 The primary purpose of the present study was to characterise changes in [HHb] response 44 dynamics in boys and girls during ramp incremental exercise in order to test the hypothesis that 45 the reduced peak $\dot{V} \mathrm{O}_{2}$ in girls is associated with a poorer matching of muscle $\mathrm{O}_{2}$ delivery to 46 muscle $\mathrm{O}_{2}$ utilisation, as evidenced by a more rapid increase in [HHb]. 


\section{METHODS}

\section{$48 \quad$ Participants and anthropometry}

49 In total, 31 boys (mean \pm SD age $9.9 \pm 0.3$ years) and 21 girls (age $10.0 \pm 0.4$ years) participated

50 in this study. All children and their parent(s)/guardian(s) provided informed assent and consent

51 to partake in the project, which was approved by the institutional ethics committee. The children

52 were healthy, recreationally active, and showed no contraindications to exercise to exhaustion.

54 An anthropometrical evaluation was performed before the first test for all participants. Stature 55 was measured to $0.01 \mathrm{~m}$ using a Holtain stadiometer (Holtain, Crymych, Dyfed, UK) and body 56 mass was determined using Avery beam balance scales to $0.1 \mathrm{~kg}$ (Avery, Birmingham, UK).

57 Body fat percentage was determined using an air displacement plethysmograph (BodPod 2000A;

58 Life Measurement Instruments, Concord, California, US) which was initially calibrated 59 according to the manufacturer's instructions and has been validated in children (16). Lung

60 volume was measured and body fat percentage was adjusted according to Lohman's child 61 specific equation (24). Participants were asked to arrive at the laboratory in a rested and fully 62 hydrated state, at least 3 hours postprandial and to refrain from consuming caffeinated drinks in 63 the 6 hours prior to testing.

64

\section{Experimental procedures}

66 All tests took place on an electromagnetically braked cycle ergometer (Lode Excalibur Sport, 67 Groningen, The Netherlands), with appropriate adjustments made to the ergometer seat, 68 handlebar and pedal cranks for each participant. Following a 5 minute warm up at $20 \mathrm{~W}$, the 69 participant completed a ramp incremental test in which the work rate increased by $10 \mathrm{~W} \cdot \mathrm{min}^{-1}$ 
70 until volitional exhaustion. Participants were asked to maintain a pedal cadence of $70 \mathrm{rev} \cdot \mathrm{min}^{-1}$

71 throughout the test. A maximal effort was considered to have been given if, in addition to

72 subjective indications such as sweating, hyperpnea and facial flushing, there was a consistent

73 reduction in cadence despite strong verbal encouragement. Although a supra-maximal test was

74 not performed in the current study to validate the determination of $\dot{V} \mathrm{O}_{2}$ max, in our laboratory

75 this occurs in $\sim 95 \%$ of participants despite the absence of a plateau in the $\dot{V} \mathrm{O}_{2}$-work-rate profile

76 at near exhaustion (6). Nonetheless, the term peak $\dot{V} \mathrm{O}_{2}$ will be used throughout to ensure

77 erroneous conclusions with regard to a maximal effort are not made. Peak work rate was defined

78 as the work rate attained at the point of test termination.

\section{Experimental measures}

81 Throughout each test, breath-by-breath gas exchange and ventilation (Metalyser 3B Cortex,

82 Biophysik, Leipzig, Germany) and heart rate (Polar S610, Polar Electro Oy, Kempele, Finland)

83 were measured and displayed online. Prior to each test, the gas analyzers were calibrated using

84 gases of known concentration and the turbine volume transducer was calibrated using a $3 \mathrm{~L}$

85 syringe (Hans Rudolph, Kansas City, MO).

87 The oxygenation status of the right vastus lateralis muscle was monitored using a commercially

88 available NIRS system (NIRO-300; Hamamatsu Photonics K.K, Japan). This system consists of 89 an emission probe which emits four wavelengths of light (776, 826, 845 and $905 \mathrm{~nm})$ and a

90 photon detector. The intensity of incident and transmitted light was recorded continuously at 2

$91 \mathrm{~Hz}$ and used to estimate the concentration changes relative to baseline levels for oxygenated,

92 deoxygenated and total haemoglobin. The $[\mathrm{HHb}]$ signal was used as an indicator of fractional $\mathrm{O}_{2}$ 
93 extraction within the field of interrogation $(10,15,17)$. As the contribution of myoglobin to the

94 NIRS signal is currently unresolved (36) changes in $[\mathrm{HHb}]$ are considered to reflect the

95 combined concentration of deoxygenated haemoglobin and myoglobin. The skin was initially

96 cleaned and the probes placed in a rubber holder which was adhered to the skin at the midpoint

97 of the muscle. To ensure the holder and its probes remained stationary during exercise and to

98 minimise the interference of extraneous light with the near-infrared signal a bandage was

99 wrapped around the leg. The NIRS signal was zeroed with the participant at rest in a seated

100 position with the muscle stationary and relaxed.

101

102 Data Analysis

103 The gas exchange data were interpolated to $1 \mathrm{~s}$ intervals and peak $\dot{V} \mathrm{O}_{2}$ was taken as the highest

$10410 \mathrm{~s}$ stationary average during the test. The GET was determined by the V-slope method (2) as

105 the point at which carbon dioxide $\left(\dot{V} \mathrm{CO}_{2}\right)$ production began to increase disproportionately to

$106 \dot{V} \mathrm{O}_{2}$ as identified using purpose designed software developed using LabVIEW (National

107 Instruments, Newbury, UK). The location of the GET was confirmed using the ventilatory

108 equivalents for $\dot{V} \mathrm{O}_{2}$ and $\dot{V} \mathrm{CO}_{2}$.

109

110 Prior to analysis, the ramp [HHb] response dynamics were averaged in $5 \mathrm{~s}$ bins and expressed

111 from $0 \%$ (mean from the 5 min of baseline pedalling at $20 \mathrm{~W}$ ) to $100 \%$ (the highest $5 \mathrm{~s}[\mathrm{HHb}$ ]

112 achieved during the test). The $[\mathrm{HHb}]$ response dynamics were expressed in relation to work rate

113 (W) and $\dot{V} \mathrm{O}_{2}$ in both absolute and relative terms. In line with previous research $(27,28)$, the

$114 \dot{V} \mathrm{O}_{2}$ response profile was back-shifted by $20 \mathrm{~s}$ in an attempt to account for the phase I-II, muscle

115 to lung transit time. To determine the most appropriate approach to characterise the profile of the 
$116 \% \Delta[\mathrm{HHb}]$ response (as a function of $\%$ peak work rate or $\dot{V} \mathrm{O}_{2}$ ), two models were compared

117 (GraphPad Prism 5). First, the entire $\% \Delta[\mathrm{HHb}]$ response was modelled from the onset of the

118 ramp exercise until exercise cessation using a sigmoid function $(8,12,26)$ :

$119 Y=a /\left(1+\exp ^{-(-c+d x)}\right)$

120 where $a$ represents the baseline corrected amplitude and $c$ is a constant dependent upon $d$ (the

121 slope of the sigmoid) whereby $c / d$ reveals the $x$ value that yields $50 \%$ of the total amplitude. The

122 point at which a plateau occurred in the $[\mathrm{HHb}]$ response was determined as the point at which the

$123[\mathrm{HHb}]$ response reached the lower boundary of the $95 \%$ confidence interval for the $a$ parameter.

125 Secondly, the increase in $\% \Delta[\mathrm{HHb}]$ observed throughout the middle portion of the exercise 126 protocol (beginning at the point where the $\% \Delta[\mathrm{HHb}]$ signal began a systematic increase above 127 baseline as determined visually) and the plateau which followed were characterised by a 128 piecewise function that included two linear segments (the 'double-linear model')(38). The 129 models were compared by computing the change in corrected Akaike Information Criterion 130 scores $\left(\Delta \mathrm{AIC}_{\mathrm{c}}\right)$. Contrary to previous findings in adults $(27,37)$, the sigmoid model provided a 131 superior fit in over $95 \%$ of cases according to the $\mathrm{AIC}_{\mathrm{c}}$ scores. Thus, the parameters derived 132 from the sigmoid model were used for all subsequent analyses.

134 Analysis of covariance (ANCOVA) on log transformed data was used to determine the 135 allometric relationship between body size (body mass, FFM) and $\dot{V} \mathrm{O}_{2}$ max. Common allometric 136 exponents were confirmed for all groups and power function ratios $\left(\mathrm{Y} / \mathrm{X}^{\mathrm{b}}\right)$ were computed and 137 their size-independence was checked and confirmed by performing size-residual correlations 138 against body mass and FFM. 


\section{Statistical analyses}

140 Prior to analysis, distribution normality was examined and verified using the Shapiro-Wilk test.

141 Independent samples t-tests were utilised to assess the influence of sex on the ramp test $\dot{V} \mathrm{O}_{2}$ and $142[\mathrm{HHb}]$ responses. Equality of variances was checked using Levene's test. If significant, the equal 143 variances not assumed P-value was reported. All data are presented as means \pm SD. Statistical 144 significance was accepted when $P<0.05$ and effect size (ES) statistics were used to detail the 145 magnitude of the observed effect using the mean difference and the pooled SD. An ES <0.2 was 146 trivial, $>0.2$ was small, $>0.5$ was medium and $>0.8$ was large.

148 Pearson correlation coefficients were used to assess the strength of relationships between the $149[\mathrm{HHb}]$ dynamics and peak $\dot{V} \mathrm{O}_{2}$. These correlations informed the multiple regression analyses to 150 determine the independent contribution of $[\mathrm{HHb}]$ kinetic parameters in explaining sex

151 differences in absolute peak $\dot{V} \mathrm{O}_{2}$ after accounting for other potentially important predictors (e.g. 152 sex, age, body fat \%). Initially, both sex and FFM were entered into the model given their strong 153 relationship with absolute peak $\dot{V} \mathrm{O}_{2}\left(\mathrm{~L}_{\mathrm{min}} \mathrm{m}^{-1}\right)$ in this age group (11). Subsequently, potential 154 predictor variables were considered in a stepwise manner to determine their independent 155 contribution to predicting absolute peak $\dot{V} \mathrm{O}_{2}$. Inclusion into the model was accepted with a 156 significant increase in explained variance at the 0.05 level. The adequacy of the regression model 157 was examined and verified using checks for multicollinearity (variance inflation factor, 158 tolerance) and distribution normality of the residuals.

\section{RESULTS}

161 Anthropometric characteristics were similar between boys and girls (see Table 1). 
163 The physiological responses during the ramp test to exhaustion are presented in table 2. Boys

164 achieved a higher peak $\dot{V} \mathrm{O}_{2}$ irrespective of whether expressed in absolute terms $(18.0 \%)$ or 165 relative to allometrically scaled body mass (16.2\%) or FFM (11.7\%). This was despite no sex 166 differences in maximum heart rate. The boys achieved a higher peak work-rate at exhaustion. No 167 sex difference was identified for the GET when expressed in absolute terms or relative to peak $168 \dot{V} \mathrm{O}_{2}$

\section{Ramp [HHb] response dynamics}

171 A representative profile of the modelled $[\mathrm{HHb}]$ response dynamics during ramp exercise for a 172 boy and girl participant is illustrated in figure 1 when expressed as a function of absolute and 173 relative work-rate and $\dot{V} \mathrm{O}_{2}$. The parameter estimates for the sigmoidal model are presented in

174 table 3 . When expressed against absolute work-rate boys had a higher $c / d(P=0.023, E S=0.67)$ 175 and attained a plateau at a higher work-rate $(P=0.031, E S=0.66)$. However, when expressed 176 relative to peak work-rate, no sex differences were present for all $[\mathrm{HHb}]$ response parameters (all $177 P>0.26$, all $E S<0.35)$. Plotting $[\mathrm{HHb}]$ against absolute $\dot{V} \mathrm{O}_{2}$ showed a strong trend for boys to 178 have a higher $c / d(P=0.055, E S=0.58)$ and to achieve a plateau in the response profile at a higher 179 metabolic rate $(P=0.014, E S=0.76)$. When $[\mathrm{HHb}]$ was plotted relative to $\dot{V} \mathrm{O}_{2}$ however, there 180 were no sex differences for response parameters (all $P>0.20$, all $E S<0.41$ ).

\section{Correlations between aerobic function and [HHb] response dynamics}

183 A significant correlation was evident between absolute peak $\dot{V} \mathrm{O}_{2}$ and the $[\mathrm{HHb}] c / d(r=0.62$, $184 P<0.001 ; r=0.79, P<0.001)$ and plateau $(r=0.70, P<0.001 ; r=0.77, P<0.001)$ when expressed as 
185 a function of absolute work rate and $\dot{V} \mathrm{O}_{2}$, respectively (see figure 2 for example correlations).

186 When the $[\mathrm{HHb}]$ response parameters were derived using relative work rate, similar, although

187 weaker, relationships were manifest between absolute $\dot{V} \mathrm{O}_{2}$ max and the $c / d$ parameter $(r=0.37$,

$188 P=0.009)$ and plateau $(r=0.30, P=0.035)$. No correlations were evident between peak $\dot{V} \mathrm{O}_{2}$ and

189 the $[\mathrm{HHb}]$ parameters derived using relative $\dot{V} \mathrm{O}_{2}$.

190

191 Muscle $[\mathrm{HHb}]$ response dynamics were also correlated with peak $\dot{V} \mathrm{O}_{2}$ normalised using

192 allometric models for body mass or FFM, although only the latter results are presented due to the

193 similar outcomes across body size measures. Relationships were observed between FFM

194 normalised $\dot{V} \mathrm{O}_{2} \max$ and the [HHb] $c / d(r=0.34, P=0.017$ and $r=0.52, P<0.001)$, and plateau

$195(r=0.45, P=0.001$ and $r=0.53, P<0.001)$ when expressed using absolute work rate and $\dot{V} \mathrm{O}_{2}$,

196 respectively. However, these relationships disappeared when [HHb] was expressed using relative

197 work rate and $\dot{V} \mathrm{O}_{2}$.

198

199 The FFM scaled peak $\dot{V} \mathrm{O}_{2}$ was significantly related to the absolute GET $(r=0.52, P<0.001)$

200 across the sample. When the GET was correlated against the [HHb] dynamics, a relationship was

201 found for $[\mathrm{HHb}] c / d(r=0.52, P<0.001)$ and the $[\mathrm{HHb}]$ plateau $(r=0.47, P<0.001)$ as a function

202 of absolute $\dot{V} \mathrm{O}_{2}$.

203

\section{Regression analysis of peak $\dot{V} \mathrm{O}_{2}$ determinants}

205 The output from the multiple linear regression prediction of absolute peak $\dot{V} \mathrm{O}_{2}$ is provided in 206 table 4. Model 1 initially started with sex and FFM entered into the model $\left(R^{2}=0.41, P<0.001\right)$. 
207 Subsequently stepwise regression revealed significant improvements in explained variance due 208 to the addition of absolute GET $\left(\Delta R^{2}=0.23, P<0.001\right)$, the $[\mathrm{HHb}]$ plateau expressed against

209 absolute $\dot{V} \mathrm{O}_{2}\left(\Delta R^{2}=0.12, P<0.001\right)$ and body fat $\%\left(\Delta R^{2}=0.03, P=0.034\right)$. The final model

210 predicted $\sim 81 \%$ of the change in absolute peak $\dot{V} \mathrm{O}_{2}\left(R^{2}=0.81, P<0.001\right)$.

211

\section{DISCUSSION}

213 The primary purpose of the present study was to examine whether sex-specific differences in the 214 temporal response of local muscle fractional $\mathrm{O}_{2}$ extraction, as indicated by the NIRS-derived $215 \Delta[\mathrm{HHb}]$ response, are present in children and account for the sexual dimorphism in peak $\dot{V} \mathrm{O}_{2}$. In 216 agreement with our hypothesis, when muscle $[\mathrm{HHb}]$ was expressed against absolute work rate

217 and $\dot{V} \mathrm{O}_{2}$, girls had a greater rate of change in $[\mathrm{HHb}]$ as evidenced by the lower $c / d$ parameter 218 and plateau. However, when expressed against relative work-rate or $\dot{V} \mathrm{O}_{2}$, the sex difference in $219[\mathrm{HHb}]$ response dynamics was no longer significant. Significant correlations were observed 220 between absolute and FFM normalised peak $\dot{V} \mathrm{O}_{2}$ and the $\mathrm{HHb} c / d$ and plateau parameters when 221 expressed against absolute work-rate or $\dot{V} \mathrm{O}_{2}$. Furthermore, when entered into a multiple 222 regression model, the [HHb] plateau against absolute $\dot{V} \mathrm{O}_{2}$ contributed to $\sim 12 \%$ of the variance 223 in peak $\dot{V} \mathrm{O}_{2}$ after adjusting for FFM, GET, and body fatness. These data, therefore, support the

224 hypothesis that the sex-difference in peak $\dot{V} \mathrm{O}_{2}$ in 9-10 year old children is, in part, related to 225 sex-specific changes in muscle $\mathrm{O}_{2}$ extraction dynamics during incremental exercise.

227 In accord with previous studies $(1,11,13,39)$, the magnitude of the sexual dimorphism in peak $228 \dot{V} \mathrm{O}_{2}$ of the children in the current study varied in relation to the different methods of expressing 
229 peak $\dot{V} \mathrm{O}_{2}$. Specifically, boys demonstrated a $\sim 18 \%$ higher peak $\dot{V} \mathrm{O}_{2}$ compared to girls when

230 expressed in absolute terms, which was reduced following allometric modelling using body mass

231 ( 16\% difference) and FFM ( 12\% difference). This residual difference following normalization

232 to FFM is consistent with other studies $(11,34)$. For example, in a cross-sectional study

233 consisting of 248 children aged 8-11 years, Dencker and colleagues (11) found, through multiple

234 regression, girls to have a lower peak $\dot{V} \mathrm{O}_{2}$ after accounting for differences in body composition,

235 heart size and habitual physical activity. Furthermore, previous data from our laboratory have

236 shown that after matching children for FFM, boys' maintain a $\sim 14 \%$ higher peak $\dot{V} \mathrm{O}_{2}$ despite no

237 sex-related differences in blood haemoglobin concentration, cardiac output and heart dimensions

238 (43). The authors attributed the higher peak $\dot{V} \mathrm{O}_{2}$ in boys to a greater muscle $\mathrm{O}_{2}$ extraction, as

239 evidenced by a $\sim 17 \%$ wider arterial mixed venous $\mathrm{O}_{2}$ content difference. This calculation,

240 however, was based on whole-body measures of maximal $\dot{V} \mathrm{O}_{2}$ and cardiac output via re-

241 arrangement of the Fick equation, which is unlikely to reflect the dynamics of muscle $\mathrm{O}_{2}$

242 delivery and $\mathrm{O}_{2}$ utilisation within the microcirculation of the contracting mycocytes over the

243 range of metabolic rates leading to peak $\dot{V} \mathrm{O}_{2}(28)$.

245 In the present study we used NIRS to non-invasively measure microcirculatory changes in [HHb]

246 in the vastus lateralis muscle to provide insight into changes in the rate of fractional muscle $\mathrm{O}_{2}$

247 extraction dynamics during ramp exercise. In agreement with previous studies in children (26,

$24835)$ and adults $(8,12)$, the $[\mathrm{HHb}]$ response during ramp exercise was well characterized using a

249 sigmoidal model, when compared to a bi-linear model (37). It has been suggested that under 250 conditions in which muscle $\mathrm{O}_{2}$ delivery is compromised (e.g. disease, detraining) a leftward shift 251 (i.e. more rapid increase) of the muscle [HHb] response is manifest (15). Consistent with this 
252 notion are data showing a more rapid increase in muscle [HHb] in untrained children (26) and 253 adults (8), the elderly (18) and adult women compared to men (27). In agreement with the latter

254 study, the girls in the current study were similarly characterised by a greater rate of change in $255[\mathrm{HHb}]$ during ramp exercise compared to boys. Specifically, at a given work-rate or metabolic 256 rate, the change in $[\mathrm{HHb}]$, expressed as a percentage of the total $[\mathrm{HHb}]$ amplitude, was greater in 257 girls compared to boys resulting in the earlier attainment of a plateau (i.e. maximal rate of $\mathrm{O}_{2}$ 258 extraction) in the $[\mathrm{HHb}]$ response. As the pattern of muscle $[\mathrm{HHb}]$ during ramp exercise reflects 259 the ratio of muscle $\mathrm{O}_{2}$ delivery to consumption, this finding implies that microvascular blood 260 flow (15) was reduced in girls at sub-maximal work-rates and $\dot{V} \mathrm{O}_{2}$ compared to boys, such that 261 the 'linear' portion of the muscle $\mathrm{O}_{2}$ delivery to utilisation relationship (plateau) was reached 262 earlier in the test while $\dot{V} \mathrm{O}_{2}$ was still increasing.

264 Interestingly, the current study's data cohere with a recent study showing female adolescents and 265 adults to have a shorter [HHb] time delay at the onset of high-intensity quadriceps exercise, 266 suggesting impaired muscle $\mathrm{O}_{2}$ delivery (42). However, such findings are in conflict with data 267 showing women to have an increased femoral blood flow to work-rate relationship during 268 incremental knee-extensor exercise (31), suggesting women would be characterised by a lower 269 rate of muscle $\mathrm{O}_{2}$ extraction during ramp cycling exercise in the current study. However, it 270 should be noted that while adult studies generally show women to have greater muscle perfusion 271 during exercise at similar exercise intensities compared to their male counterparts, this is 272 dependent on the type (sustained vs. intermittent) of muscle contraction and recruited muscle 273 mass (20). Compared to knee-extensor exercise, cycling exercise involves recruitment from 274 muscles across the lower limbs and is not restricted to the quadriceps (33). Thus, as highlighted 
275 by Murias et al. (27), in contrast to knee-extensor exercise the additional muscle mass recruited 276 during cycling exercise will elicit a maximal cardiac output response which needs to be

277 effectively redistributed to the metabolically active fibres. Taken collectively, our data and that

278 of Murias et al. (27) suggest that under conditions of ramp cycling exercise to exhaustion, 279 females are characterised by an impaired muscle $\mathrm{O}_{2}$ delivery in both prepubertal children and 280 young adults.

281

282 While the mechanistic basis for the more rapid rate of change in muscle [HHb] for a given work 283 rate and $\dot{V} \mathrm{O}_{2}$ in girls cannot be explained with our data, a reduction in bulk blood flow, poorer 284 regional matching of blood flow to the metabolically active mycocytes and/or lower muscle 285 oxidative capacity may be implicated. It has been suggested that the mechanical effects of 286 muscle contraction and/or localised vasodilators may play a role in altering the [HHb] dynamics 287 during ramp exercise $(8,15)$, but these factors are likely to predominate during the early portion 288 of the ramp test. Alternatively, Murias and colleagues (27) suggested that the haemodynamic 289 response in women may be compromised due to sex-specific differences in sympathetic 290 activation limiting the re-distribution of blood flow to the contracting muscles. Unfortunately, 291 complementary data on muscle blood flow at rest or during exercise in children are not available, 292 although studies have shown micro- and macro- vascular function to be sex-independent in 293 healthy children $(19,32)$. Furthermore, although limited to rest and maximal exercise, our 294 laboratory has previously reported that with boys and girls of similar FFM there is no difference 295 in cardiac dimensions, stroke volume and cardiac output (43). Muscle oxidative capacity is likely 296 to be an important determinant of the muscle $[\mathrm{HHb}]$ response, but no data are available on sex297 differences in muscle oxidative enzyme activates in pediatric groups. In contrast, the recovery of 
muscle PCr following exercise can be used as a non-invasive index of the muscles oxidative

299 capacity and is not sex-dependent in prepubertal children (4). Alternatively, it is plausible that

300 sex-differences in the progressive recruitment of higher-order muscle fibres during ramp exercise

301 may account for the more rapid increase in muscle [HHb] in girls. Specifically, it has been

302 shown that type II fibres with a low oxidative capacity are characterised by more rapid muscle

$303 \mathrm{O}_{2}$ extraction kinetics at the onset of muscle contractions, presumably due to sluggish muscle $\mathrm{O}_{2}$

304 delivery dynamics relative to muscle $\mathrm{O}_{2}$ consumption $(7,25)$. While, muscle fibre recruitment

305 patterns remain to be elucidated during exercise in children, it is pertinent to note that girls are

306 characterised by slower $\dot{V} \mathrm{O}_{2}$ kinetics during cycling exercise (14) and a greater muscle

307 metabolic perturbation (e.g. PCr breakdown) during high-intensity incremental (5) or

308 squarewave (42) exercise, which may be indicative of a greater reliance on higher-order muscle

309 fibres and reduced muscle $\mathrm{O}_{2}$ availability. Although not definitive, this suggests that sex-

310 differences in the progressive recruitment of type II muscle fibres during ramp exercise may

311 explain, in part, our observation of more rapid [HHb] kinetics in girls. However, it should be

312 noted, that such sex-differences in muscle phosphate and $\mathrm{pH}$ responses are not seen during high-

313 intensity intermittent exercise in children (22) or adolescents (41), suggesting muscle blood flow

314 may not be compromised in females under such experimental conditions and that the findings of

315 the current study reflect the incremental exercise protocol employed.

317 In order to determine whether the changes in muscle [HHb] dynamics accounted for the sex-

318 differences in peak $\dot{V} \mathrm{O}_{2}$ in the current study, multiple regression analyses were performed. After

319 adjusting for FFM, the model predicted $\sim 81 \%$ of the variance in absolute peak $\dot{V} \mathrm{O}_{2}$ and

320 revealed significant contributions from the GET, muscle [HHb] plateau and percentage body fat. 
321 In particular, the muscle $[\mathrm{HHb}]$ plateau (derived relative to absolute $\dot{V} \mathrm{O}_{2}$ ) accounted for $\sim 12 \%$

322 of the explained variance and rendered the sex term non-significant. This indicates that sex

323 differences in peak $\dot{V} \mathrm{O}_{2}$ can be explained, in part, by muscle $\mathrm{O}_{2}$ delivery to muscle $\mathrm{O}_{2}$ utilisation

324 dynamics. The model derived from the present study explains a greater percentage of the

325 variance in peak $\dot{V} \mathrm{O}_{2}$ than previously reported in children by others $(11,30)$. Interestingly, in the

326 present study, FFM (and sex) accounted for $\sim 41 \%$ of the variance in absolute peak $\dot{V} \mathrm{O}_{2}$ which

327 is strikingly comparable to previous studies, and presumably accounts for cardiac function and

328 morphology in our participants, although this was not directly measured. The present study

329 extends this observation by demonstrating that an additional $\sim 40 \%$ of the variance for predicting

330 peak $\dot{V} \mathrm{O}_{2}$ was attributed to the GET and [HHb] plateau, as percentage body fat only improved

331 the model by $\sim 3 \%$. To our knowledge, the GET and [HHb] dynamics have not been considered

332 in previous work concerning the determinants of peak $\dot{V} \mathrm{O}_{2}$ in children and is likely to reflect

333 differences in the participants' muscle oxidative capacity and muscle fibre distribution as both

334 the GET (21) and muscle [HHb] responses (as discussed above) are influenced by these factors.

336 Although hypothesised in initial modelling simulations (15), Boone et al. (8) were the first to

337 demonstrate a relationship between muscle $[\mathrm{HHb}]$ dynamics during ramp exercise and peak $\dot{V} \mathrm{O}_{2}$

338 in adult cyclists and physically active students. Subsequently, McNarry et al. (26) demonstrated a

339 relationship between muscle $[\mathrm{HHb}] c / d$ and parameters of aerobic function (peak $\dot{V} \mathrm{O}_{2}$ and GET)

340 in girls during cycling exercise. Similar to previous findings in adults and children, in the present

341 study we observed a positive relationship between the [HHb] response dynamics (c/d, plateau)

342 and peak $\dot{V} \mathrm{O}_{2}$ (expressed in absolute terms or scaled for FFM) and submaximal (GET)

343 parameters of aerobic function. This supports the putative role of aerobic conditioning on 
344 causing a 'rightward' shift in the [HHb] response, and is likely to reflect enhanced muscle

345 oxidative capacity and muscle fibre type distribution $(8,26)$. However, an interesting finding in

346 the current study is that the sex differences in muscle [HHb] dynamics ( $c / d$ and plateau)

347 disappeared when expressed relative to peak work rate and $\dot{V} \mathrm{O}_{2}$. Both absolute peak $\dot{V} \mathrm{O}_{2}$ and 348 peak work-rate were lower in girls in the current study, meaning that expressing [ $\mathrm{HHb}]$ at any

349 given $\dot{V} \mathrm{O}_{2}$ or work-rate would represent a greater proportion of their peak response. Similar

350 findings have been reported when comparing younger and older adults (18) and males and

351 females (27), although the differences persisted when expressed relative to peak $\dot{V} \mathrm{O}_{2}$ in the latter 352 study.

354 It is prudent to note certain limitations with the present study design. Specifically, although 355 chronological age of the participants in the current study is comparable with previous studies $356(11,39,43)$ and suggests our group were pre-pubertal, this was not determined. Unfortunately, 357 the ethical considerations that surround the utilization of Tanner stages or skeletal age and the 358 inaccuracy associated with age to peak height velocity make the accurate determination of 359 maturity stage challenging. Furthermore, no central measures of bulk $\mathrm{O}_{2}$ delivery or 360 haemoglobin were collected in the present study, although normalization by FFM has previously 361 been shown to account for differences in these parameters between the sexes (39). Habitual 362 physical activity or participation in structured sports was not measured in the current study. 363 However, after accounting for body size and cardiac dimensions, physical activity (specifically 364 vigorous physical activity) only accounts for $\sim 1 \%$ of the explained variance in peak $\dot{V} \mathrm{O}_{2}$ in pre365 pubertal boys and girls (11). Furthermore, a recent review highlighted that there is no meaningful 366 evidence of a relationship between children's habitual physical activity and aerobic fitness as 
367 expressed by peak $\dot{V} \mathrm{O}_{2}(2)$, suggesting sex-differences in habitual physical activity are unlikely

368 to be a confounding factor in the current study's findings. Finally, the interpretation of the [HHb]

369 kinetics obtained by NIRS requires particular methodological considerations, including i)

370 variations in adiposity beneath the probe between boys and girls; ii) the generalizability of the

371 response dynamics from a localised area to a heterogeneous muscle and iii) the [HHb] response

372 has been shown to be influenced by muscle activation patterns (9). The absence of EMG

373 measures from the present study precludes the possibility that sex differences in muscle activity

374 may explain the altered $[\mathrm{HHb}]$ response from being excluded. However, it is important to

375 recognize that there were no differences in FFM between sexes in the current study and changes

376 in $[\mathrm{HHb}]$ were normalized to the peak value at exhaustion. Furthermore, the NIRS probe was

377 placed in the same location for all participants, minimizing regional differences.

379 CONCLUSION

380 In conclusion, this is the first study to utilise NIRS derived changes in the muscle [HHb]

381 response dynamics to assess the sexual dimorphism in the peak $\dot{V} \mathrm{O}_{2}$ of boys and girls. In accord

382 with our hypothesis, girls were shown to require a greater fractional $\mathrm{O}_{2}$ extraction to increase

383 work rate and $\dot{V} \mathrm{O}_{2}$ and thus reached an earlier plateau in $\mathrm{O}_{2}$ extraction compared to boys during

384 ramp exercise. Parameters of the muscle $[\mathrm{HHb}]$ dynamics were related to aerobic function and

385 the plateau in muscle [HHb] was found to account for $\sim 12 \%$ of the variance in peak $\dot{V} \mathrm{O}_{2}$ after

386 adjusting for FFM, GET and body fatness, and eliminated the sex difference in peak $\dot{V} \mathrm{O}_{2}$. These

387 results may reflect an inferior bulk $\mathrm{O}_{2}$ delivery and/or regional matching of $\mathrm{O}_{2}$ delivery in girls. 
391 The present study does not engender any conflict of interests and does not constitute an 392 endorsement by ACSM.

4. Barker AR, Welsman JR, Fulford J, Welford D, Armstrong N. Muscle phosphocreatine kinetics in children and adults at the onset and offset of moderate-intensity exercise. J Appl Physiol. 2008;105(2):446-56.

5. Barker AR, Welsman JR, Fulford J, Welford D, Armstrong N. Quadriceps muscle energetics during incremental exercise in children and adults. Med Sci Sports Exerc. 2010;42(7):1303-13.

6. Barker AR, Williams CA, Jones AM, Armstrong N. Establishing maximal oxygen uptake in young people during a ramp cycle test to exhaustion. Br J Sports Med. 2011;45:498-503.

7. Behnke BJ, McDonough P, Padilla DJ, Musch TI, Poole DC. Oxygen exchange profile in rat muscles of contrasting fibre types. $J$ Physiol. 2003;549(2):597-605.

8. Boone J, Koppo K, Barstow TJ, Bouckaert J. Pattern of deoxy[Hb+Mb] during ramp cycle exercise: influence of aerobic fitness status. Eur J Appl Physiol. 2009;105(6):851-9.

9. Chin LMK, Kowalchuk JM, Barstow TJ et al. The relationship between muscle deoxygenation and activation in different muscles of the quadriceps during cycle ramp exercise. J. Appl. Physiol. 2011;111(5):1259-65.

10. DeLorey DS, Kowalchuk JM, Paterson DH. Relationship between pulmonary $\mathrm{O}_{2}$ uptake kinetics and muscle deoxygenation during moderate-intensity exercise. J. Appl. Physiol. 2003;95(1):11320.

11. Dencker M, Thorsson O, Karlsson MK et al. Gender differences and determinants of aerobic fitness in children aged 8-11 years. Eur J Appl Physiol. 2007;99(1):19-26.

421 12. DiMenna FJ, Bailey SJ, Jones AM. Influence of body position on muscle deoxy[Hb+Mb] during ramp cycle exercise. Respir Physiol Neurobiol. 2010;173(2):138-45. 
13. Eiberg S, Hasselstrom H, Gronfeldt V, Froberg K, Svensson J, Andersen LB. Maximum oxygen

14. Fawkner SG, Armstrong N. Sex differences in the oxygen uptake kinetic response to heavyuptake and objectively measured physical activity in Danish children 6-7 years of age: the Copenhagen school child intervention study. Br J Sports Med. 2005;39(10):725-30.

15. Ferreira LF, Koga S, Barstow TJ. Dynamics of noninvasively estimated microvascular $\mathrm{O}_{2}$ intensity exercise in prepubertal children. Eur J Appl Physiol. 2004;93(1-2):210-6.

16. Fields DA, Goran MI, McCrory MA. Body-composition assessment via air-displacement extraction during ramp exercise. J. Appl. Physiol. 2007;103(6):1999-2004.

17. Grassi B, Pogliaghi S, Rampichini S et al. Muscle oxygenation and pulmonary gas exchange plethysmography in adults and children: a review. Am J Clin Nutr. 2002;75(3):453-67.

18. Gravelle BM, Murias JM, Spencer MD, Paterson DH, Kowalchuk JM. Adjustments of pulmonary $\mathrm{O} 2$ uptake and muscle deoxygenation during ramp incremental exercise and constant-load moderate-intensity exercise in young and older adults. J Appl Physiol 2012;113(9):1466-75. 19. Hopkins ND, Stratton G, Tinken TM et al. Relationships between measures of fitness, physical
activity, body composition and vascular function in children. Atherosclerosis. 2009;204(1):244-9.

20. Hunter SK. Sex differences in human fatigability: mechanisms and insight to physiological responses. Acta Physiol. 2014;210(4):768-89.

21. Jones AM, Carter H. The effect of endurance training on parameters of aerobic fitness. Sports Med. 2000;29(6):373-86.

22. Kappenstein J, Ferrauti A, Runkel B, Fernandez-Fernandez J, Muller K, Zange J. Changes in phosphocreatine concentration of skeletal muscle during high-intensity intermittent exercise in children and adults. Eur J Appl Physiol. 2013;113(11):2769-79.

23. Koga S, Kano Y, Barstow TJ et al. Kinetics of muscle deoxygenation and microvascular PO(2) during contractions in rat: comparison of optical spectroscopy and phosphorescence-quenching techniques. J Appl Physiol. 2012;112(1):26-32.

24. Lohman TG. Assessment of Body Composition in Children. Pediatr. Exerc. Sci. 1986;1:19-30.

25. McDonough P, Behnke BJ, Padilla DJ, Musch TI, Poole DC. Control of microvascular oxygen pressures in rat muscles comprised of different fibre types. J Physiol. 2005;563(3):903-13.

26. McNarry MA, Welsman JR, Jones AM. Influence of training and maturity status on the cardiopulmonary responses to ramp incremental cycle and upper body exercise in girls. J Appl Physiol. 2011;110(2):375-81.

27. Murias JM, Keir DA, Spencer MD, Paterson DH. Sex-related differences in muscle deoxygenation during ramp incremental exercise. Respir. Physiol. Neuro. 2013;189(3):530-6. 
28. Murias JM, Spencer MD, Keir DA, Paterson DH. Systemic and vastus lateralis muscle blood flow and $\mathrm{O} 2$ extraction during ramp incremental cycle exercise. Am J Physiol Regul Integr Comp Physiol. 2013;304(9):R720-5.

460 29. Obert P, Mandigout S, Nottin S, Vinet A, N'Guyen LD, Lecoq AM. Cardiovascular responses to

30. Obert P, Mandigout S, Vinet A, Nottin S, N'Guyen LD, Lecoq AM. Relationships between left

31. Parker BA, Smithmyer SL, Pelberg JA, Mishkin AD, Herr MD, Proctor DN. Sex differences in

37. Spencer M, Murias J, Paterson D. Characterizing the profile of muscle deoxygenation during

38. Vieth E. Fitting piecewise linear regression functions to biological responses. $J$ Appl Physiol.

32. Radtke T, Khattab K, Eser P, Kriemler S, Saner H, Wilhelm M. Puberty and microvascular leg vasodilation during graded knee extensor exercise in young adults. J Appl Physiol (1985). 2007;103(5):1583-91.

33. Richardson RS, Frank LR, Haseler LJ. Dynamic knee-extensor and cycle exercise: functional MRI of muscular activity. Int J Sports Med. 1998;19(3):182-7.

34. Rowland T, Goff D, Martel L, Ferrone L. Influence of cardiac functional capacity on gender differences in maximal oxygen uptake in children. Chest. 2000;117(3):629-35.

35. Saynor ZL, Barker AR, Oades PJ, Williams CA. Impaired Aerobic Function in Young Cystic Fibrosis Patients During Ramp Exercise. Med Sci Sports Exerc. 2014.

36. Seiyama A, Hazeki O, Tamura M. Noninvasive quantitative analysis of blood oxygenation in rat skeletal muscle. J Biochem. 1988;103(3):419-24.

39. Vinet A, Mandigout S, Nottin S et al. Influence of body composition, hemoglobin concentration, and cardiac size and function of gender differences in maximal oxygen uptake in prepubertal children. Chest. 2003;124(4):1494-9.

40. Welsman JR, Armstrong N, Kirby BJ, Winsley RJ, Parsons G, Sharpe P. Exercise performance and magnetic resonance imaging determined thigh muscle volume in children. Eur. J. Appl. Physiol. 1997;76:92-7.

41. Willcocks RJ, Fulford J, Armstrong N, Barker AR, Williams CA. Muscle metabolism during fatiguing isometric quadriceps exercise in adolescents and adults. Appl Physiol Nutr Metab. 2014;39(4):439-45.

42. Willcocks RJ, Williams CA, Barker AR, Fulford J, Armstrong N. Age- and sex-related differences in muscle phosphocreatine and oxygenation kinetics during high-intensity exercise in adolescents and adults. NMR Biomed. 2010;23(6):569-77. 
43. Winsley RJ, Fulford J, Roberts AC, Welsman JR, Armstrong N. Sex difference in peak oxygen uptake in prepubertal children. J. Sci. Med. Sport. 2009;12(6):647-51.

\section{FIGURE CAPTION}

Figure 1. Deoxygenated haemoglobin plus myoglobin concentration ([HHb]) response as a function of a) absolute work rate (WR), b) relative work rate, c) absolute $\dot{V} \mathrm{O}_{2}$, and d) relative $\dot{\mathrm{VO}}_{2}$ for a representative boy (०) and $\operatorname{girl}(\bullet)$. 
Figure 2. The relationship between absolute peak $\dot{V} \mathrm{O}_{2}$ and muscle $[\mathrm{HHb}] \mathrm{c} / \mathrm{d}(\mathrm{A})$ and plateau (B) as a function of absolute $\dot{V} \mathrm{O}_{2}$ in boys (०) and girls $(\bullet)$. Results for the Pearson's correlation are presented. See text for further details. 